\title{
Differential regulation of claudin-2 and claudin-15 expression in children and adults with malabsorptive disease
}

\author{
M. Lora D. M. Ong ${ }^{1} \cdot$ Sunil Yeruva $\mathbb{C}^{1,2} \cdot$ Anne Sailer $^{2} \cdot$ Steven P. Nilsen ${ }^{1} \cdot$ Jerrold R. Turner $\mathbb{C}^{1,2}$
}

Received: 23 March 2018 / Revised: 20 August 2019 / Accepted: 4 September 2019 / Published online: 11 October 2019

(c) The Author(s), under exclusive licence to United States and Canadian Academy of Pathology 2019

\begin{abstract}
Intestinal $\mathrm{Na}^{+}$-nutrient cotransport depends on claudin- 2 and claudin- 15 mediated $\mathrm{Na}^{+}$recycling. Expression of these proteins is coordinately regulated during postnatal development. While expression of claudin- 2 and claudin- 15 has been studied in inflammatory bowel disease (IBD) and celiac disease (CD), it has not been assessed in other malabsorptive diseases, and no reports have compared expression in children and adults. We used quantitative immunofluorescence microscopy to assess claudin-2 and claudin-15 expression in duodenal biopsies from children and adults with malabsorptive disease and healthy controls. Consistent with previous work in rodents, claudin-2 expression in healthy children was markedly greater, and claudin- 15 expression was less, than that in adults. Claudin-2 expression was increased in adults with CD and downregulated in children with graft-versus-host disease (GVHD). In contrast, claudin-15 expression was reduced in adults with GVHD and common variable immunodeficiency (CVID). These data show that one of the two $\mathrm{Na}^{+}$/water pore-forming claudins is upregulated in CD and downregulated in GVHD and CVID. The specific claudin whose expression changes, however, reflects the age of the patient (child or adult). We conclude that contributions of claudin-2 and claudin-15 to pathophysiology of and responses to diarrhea in children and adults with GVHD and CVID differ from those in $\mathrm{CD}$ and IBD.
\end{abstract}

\section{Introduction}

The tight junction proteins claudin-2 and claudin-15 have been identified as essential regulators of paracellular $\mathrm{Na}^{+}$ flux and transcellular nutrient absorption [1, 2]. Tsukita et al. showed that knockout of claudin-2 alone does not have a marked effect on intestinal transport [1,3], but that claudin-15 knockout causes intestinal hypertrophy [4]. One might therefore hypothesize that knockout of both claudin-2 and claudin-15 would result in a phenotype similar to claudin-15 knockout. However, double knockout mice died

These authors contributed equally: M. Lora D. M. Ong, Sunil Yeruva

Jerrold R. Turner

jrturner@bwh.harvard.edu

1 Laboratory of Mucosal Barrier Pathobiology, Department of Pathology, Brigham and Women's Hospital and Harvard Medical School, Boston, MA, USA

2 Department of Pathology, The University of Chicago, Chicago, IL, USA of within 3 weeks of birth as a result of inadequate $\mathrm{Na}^{+}$dependent nutrient transport, which mediates absorption of most nutrients $[2,5,6]$. This was due to reduced $\mathrm{Na}^{+}$within the gut lumen rather than loss of $\mathrm{Na}^{+}$-nutrient cotransporters. Malabsorptive death follows because normal diets do not contain sufficient $\mathrm{Na}^{+}$to drive absorption. $\mathrm{Na}^{+}$must therefore be recycled from the lamina propria back to the gut lumen in order to support additional rounds of transcellular transport. This $\mathrm{Na}^{+}$recycling depends on flux across paracellular $\mathrm{Na}^{+}$channels formed from claudin-2 and claudin- 15 .

Consistent with their essential roles, expression of claudin-2 and claudin- 15 is tightly regulated during development [7]. Claudin-2 is highly expressed at birth and is rapidly down regulated thereafter [7]. Conversely, at birth claudin-15 is only expressed at low levels, but is markedly upregulated after weaning. It therefore appears that claudin15 supplants claudin-2 function during postnatal intestinal development. While the low levels of claudin- 15 expressed are sufficient to allow claudin-2 knockout mice to survive the neonatal period, the limited claudin- 2 expression in adults is insufficient to support nutrient absorption in claudin-15 knockout mice [1]. The intestinal hypertrophy 
that occurs in claudin-15 knockout mice can therefore be understood as a physiologic adaptation whereby increases in mucosal surface area compensate for inefficient nutrient and water absorption. Finally, the observation that knockout of both claudin-2 and claudin-15 leads to malabsorptive death indicates that these are the only claudin proteins that can adequately support $\mathrm{Na}^{+}$recycling and nutrient absorption.

Previous studies have demonstrated that claudin-2 expression is upregulated in inflammatory bowel disease (IBD) [8, 9] or celiac disease (CD) [9-11]. Similarly, one study has suggested that claudin-15 is modestly upregulated in CD [10]. Claudin-2 and claudin-15 upregulation in these malabsorptive diseases may be a physiologic response representing efforts to increase absorption to compensate for reduced mucosal surface area. We therefore hypothesized that claudin-2 and claudin-15 expression might be increased in other malabsorptive diseases of the small intestine.

To better understand the pathophysiology of malabsorption and barrier dysfunction in disease, we assessed claudin-2 and claudin-15 expression in three diarrheal diseases. The first two, CD [12] and graft-versus-host disease (GVHD) [13-15], are characterized by increased intestinal permeability as well as malabsorptive diarrhea. The third, common variable immunodeficiency (CVID) is less welldefined and has not been studied in terms of intestinal permeability or tight junction protein expression.

$\mathrm{CD}$ is an immune-mediated disorder [16-18], in which dietary gluten-derived peptides trigger epithelial stress, immune activation, and $\mathrm{T}$ cell-mediated epithelial damage in susceptible individuals [19-21]. This reduces epithelial survival and results in accelerated epithelial turnover that leads to villus atrophy, reduced and absorptive surface area, and loss of fully mature enterocytes, as their rapid turnover does not permit complete differentiation [17, 18].

GVHD occurs as a complication of hematopoietic stem cell (bone marrow) transplantation [22, 23]. Similar to CD, GVHD patients suffer from malabsorptive diarrhea [24], which, in many cases, is associated with villous atrophy [24]. The hallmark of GVHD is, however, crypt epithelial cell apoptosis due to direct attack by CD8+T cells [24, 25]. As with CD, diarrhea in GVHD is thought to reflect loss of both absorptive surface area and mature enterocytes. Despite these similarities, pathogenesis of CD and GVHD are quite different from one another.

Although rare, CVID is one of the most common primary immunodeficiencies, with a prevalence of 1 case per 10,000-250,000 individuals, and results from a generalized defect in immunoglobulin synthesis [26]. While the majority of cases are sporadic, a variety of genetic defects, primarily within the immune system, have been identified in familial CVID, which comprises $\sim 10 \%$ of patients. CVID typically presents in the third and fourth decades of life, and $\sim 25 \%$ of patients suffer from autoimmune disease. The histologic features of small bowel disease in CVID vary and can overlap significantly with both CD and GVHD [27]. While many patients present with diarrhea and malabsorptive disease, the mechanisms are not well defined.

The aim of this study was to assess expression of the $\mathrm{Na}^{+}$and water pore-forming claudins, claudin- 2 and claudin-15, in children and adults with malabsorptive disease. We used duodenal biopsies from patients and age-matched healthy subjects to assess intestinal epithelial claudin-2 and claudin-15 expression.

\section{Materials and methods}

\section{Ethics statement and tissue collection}

Institutional approval was granted by the University of Chicago and Brigham and Women's Hospital Institutional Review Boards. Data from this study are not available in a public repository but will be made available upon request. Paraffin embedded duodenal biopsies were obtained from pathology department archives. A computer search identified children and adults with diagnoses of normal (within normal limits), CD, GVHD, and CVID (adults only). After examination of clinical records, pathology reports, and haematoxylin and eosin-(H\&E) stained slides, cases were arranged in high density tissue microarrays using an ATA27 automated tissue arrayer (Beecher Instruments).

\section{Immunohistochemical staining}

Claudin-2 and claudin-15 immunostains were performed on $5 \mu \mathrm{m}$ sections of formalin-fixed paraffin embedded duodenal biopsies. Slides were baked overnight at $60^{\circ} \mathrm{C}$, washed thrice in xylene, transferred to $100 \%$ ethanol, and rehydrated through graded ethanol solutions. Antigen retrieval was performed for $15 \mathrm{~min}$ in a pressure cooker using Dako Target Retrieval Solutions S1699 and S2367 (Agilent Technologies, Santa Clara, CA). After cooling on ice followed by a wash in PBS, tissues were photobleached (under bright light) in $4.5 \%$ hydrogen peroxide, $0.005 \mathrm{~N} \mathrm{NaOH}$ in PBS. The solution was replenished after $1 \mathrm{~h}$ for a total photobleaching time of $2 \mathrm{~h}$. After a brief wash in PBS, slides were incubated in blocking buffer (DAKO X090930) for $10 \mathrm{~min}$. Primary antibodies were prepared in antibody diluent (DAKO SB302283) and applied overnight at room temperature. Primary antibodies were: rabbit polyclonal anti-claudin-15, $0.25 \mu \mathrm{g} / \mathrm{mL}$ (Invitrogen 38-9200; lot\# QJ228299); rabbit polyclonal anticlaudin-2, $0.25 \mu \mathrm{g} / \mathrm{mL}$ (Atlas Antibodies HPA05154; lot\# R61400); mouse monoclonal anti-E-cadherin, $0.83 \mu \mathrm{g} / \mathrm{mL}$ (Abcam ab76055, clone M168, lot\# GR317373-12). After 
three washes in PBS, secondary antibodies were diluted as above with $10 \mu \mathrm{g} / \mathrm{mL}$ Secondary antibodies were: AlexaFluor 594 AffiniPure donkey anti-rabbit $\mathrm{IgG}, 1.5 \mu \mathrm{g} / \mathrm{mL}$ (Jackson ImmunoResearch 711- 586-152; lot\# 136184) and AlexaFluor 488 AffiniPure donkey anti-mouse $\mathrm{IgG}, 1.5 \mu \mathrm{g} / \mathrm{mL}$ (Jackson ImmunoResearch 715-545-151; lot\# 118237). These were combined with Hoescht 33342 (Thermo Fisher Scientific) and applied for $1 \mathrm{~h}$. Slides were then washed three times in PBS, dipped in water, and mounted using ProLong Diamond antifade reagent (Thermo Fisher Scientific).

\section{Quantification of claudin-2 and 15 staining intensities}

Claudin staining intensity was scored in crypt regions using Metamorph 7.8. From each tissue biopsy, a blinded observer selected three representative crypt regions on the basis of orientation, representative histology, image quality, and staining typical of the biopsy. Regions of interest were drawn to encompass $\sim 2 \mu \mathrm{m}$ of intracellular and extracellular space on either side of the apical membrane. After applying a unique threshold to exclude nonjunctional staining, the mean intensity was calculated for each region. The average of the three measurements, less the threshold, was used as a single intensity score for each biopsy. These were normalized to the mean intensity of biopsies from healthy adults.

\section{Statistical analysis}

All statistical analyses used ANOVA (GraphPad Prism 5.0). Figures show one point for each subject and 95\% confidence intervals for each group.

\section{Results}

\section{Subjects and histopathology}

We assessed duodenal biopsies from pediatric and adult subjects with normal histology, CD, active GVHD, or CVID (Table I). Pediatric cases were analyzed separately, as studies in mice suggest that claudin- 2 and claudin- 15 expression would be increased and decreased, respectively, in young children. Because those mouse studies compared mice before and after weaning, we limited the pediatric population to children under 5 years of age.

Histopathological analysis of CD patient biopsies showed typical features in both pediatric and adult cases. All patients with $C D$ had either partial or total villus atrophy and were characterized by marked intraepithelial lymphocytosis. Crypt hyperplasia was modest. Dense lamina propria lymphoplasmacytic infiltrates were also present in all
Table I Patient demographics

\begin{tabular}{|c|c|c|c|c|}
\hline & Normal & $\mathrm{CD}$ & GVHD & CVID \\
\hline & \multicolumn{4}{|c|}{ Mean age (range) years $n(\mathrm{~F}, \mathrm{M})$} \\
\hline Children & $\begin{array}{l}1.2(1-3) 15 \\
\text { F, } 23 \mathrm{M}\end{array}$ & $\begin{array}{l}1.7(1-5) \\
5 \mathrm{~F}, 9 \mathrm{M}\end{array}$ & $\begin{array}{l}2.3(1-4) \\
1 \mathrm{~F}, 5 \mathrm{M}\end{array}$ & NA \\
\hline Adults & $\begin{array}{l}51(30-80) \\
10 \mathrm{~F}, 5 \mathrm{M}\end{array}$ & $\begin{array}{l}49 \text { (27-76) } \\
13 \mathrm{~F}, 3 \mathrm{M}\end{array}$ & $\begin{array}{l}52(26-70) \\
9 \mathrm{~F}, 8 \mathrm{M}\end{array}$ & $\begin{array}{l}53(22-72) \\
3 \mathrm{~F}, 7 \mathrm{M}\end{array}$ \\
\hline
\end{tabular}

CD cases (Fig. 1). Apoptotic crypt epithelial cells were not seen.

GVHD cases were characterized by partial villus atrophy. The lamina propria was sparsely populated by immune cells and appeared somewhat fibrotic in most cases. Varying numbers of apoptotic crypt epithelial cells were present in all cases and tended to correlate with the degree of crypt hyperplasia, which ranged from mild to marked (Fig. 1). Intraepithelial lymphocytes were not prominent in any cases.

The CVID biopsies studied here ranged from nearly normal appearing histology to histopathology similar to advanced $\mathrm{CD}$. The latter included total villous atrophy, moderate crypt hyperplasia, and marked intraepithelial lymphocytosis. These cases tended to have a dense lamina propria lymphocytic infiltrate. Neither plasma cells nor apoptotic bodies were seen in any of the CVID biopsies.

\section{Claudin-2}

Claudin-2 expression in adult cases was limited to the crypt region. Although claudin-2 expression could be detected in villous enterocytes of control pediatric biopsies, villi were absent or substantially blunted in the CD and GVHD cases. Our scoring algorithm therefore focused on crypt epithelial cells. Only well-oriented crypts were studied. This allowed quantitative analysis of fluorescent intensity at intercellular junctions, i.e., tight junctions, which were easily recognized as bright punctae (Fig. 2).

Claudin-2 expression in healthy children was 3.3-fold of that in healthy adults $(P<0.005$; Fig. 3$)$. Moreover, claudin2 was highly expressed in Brunner's glands of children and adults.

Consistent with a previous report [10], we detected a $68 \%$ increase in tight junction-associated claudin-2 within duodenal biopsies of adult $\mathrm{CD}$ patients $(P<0.005$; Fig. 3$)$. We did not, however, detect any change in tight junctionassociated claudin-2 expression when pediatric CD cases and healthy controls were compared. Nevertheless, claudin2 expression in healthy and $\mathrm{CD}$ pediatric subjects remained far greater than in adult $\mathrm{CD}$ patients (2.6-fold; $P<0.001$; Fig. 3).

Disease-induced changes in claudin-2 expression also differed between pediatric and adult GVHD patients. 
Fig. 1 Duodenal biopsy histology. Representative H\&E images for each subject group are shown. White arrows indicate intraepithelial lymphocytes in CD biopsies and apoptotic crypt epithelial cells in GVHD. Note the absence of plasma cells in CVID. Scale bar $=100 \mu \mathrm{m}$; Brunner's gland scale bar $=250 \mu \mathrm{m}$

Fig. 2 Claudin-2 expression. Representative claudin-2 immunofluorescence images for each subject group are shown. Immunofluorescence shows claudin-2 (green), E-cadherin (red), and DNA (blue). Note the intense claudin-2 expression within Brunner's gland epithelia. Scale bar $=100 \mu \mathrm{m}$; inset bar $=$ $20 \mu \mathrm{m}$. Brunner's gland scale: bar $=250 \mu \mathrm{m}$; inset bar $=50 \mu \mathrm{m}$
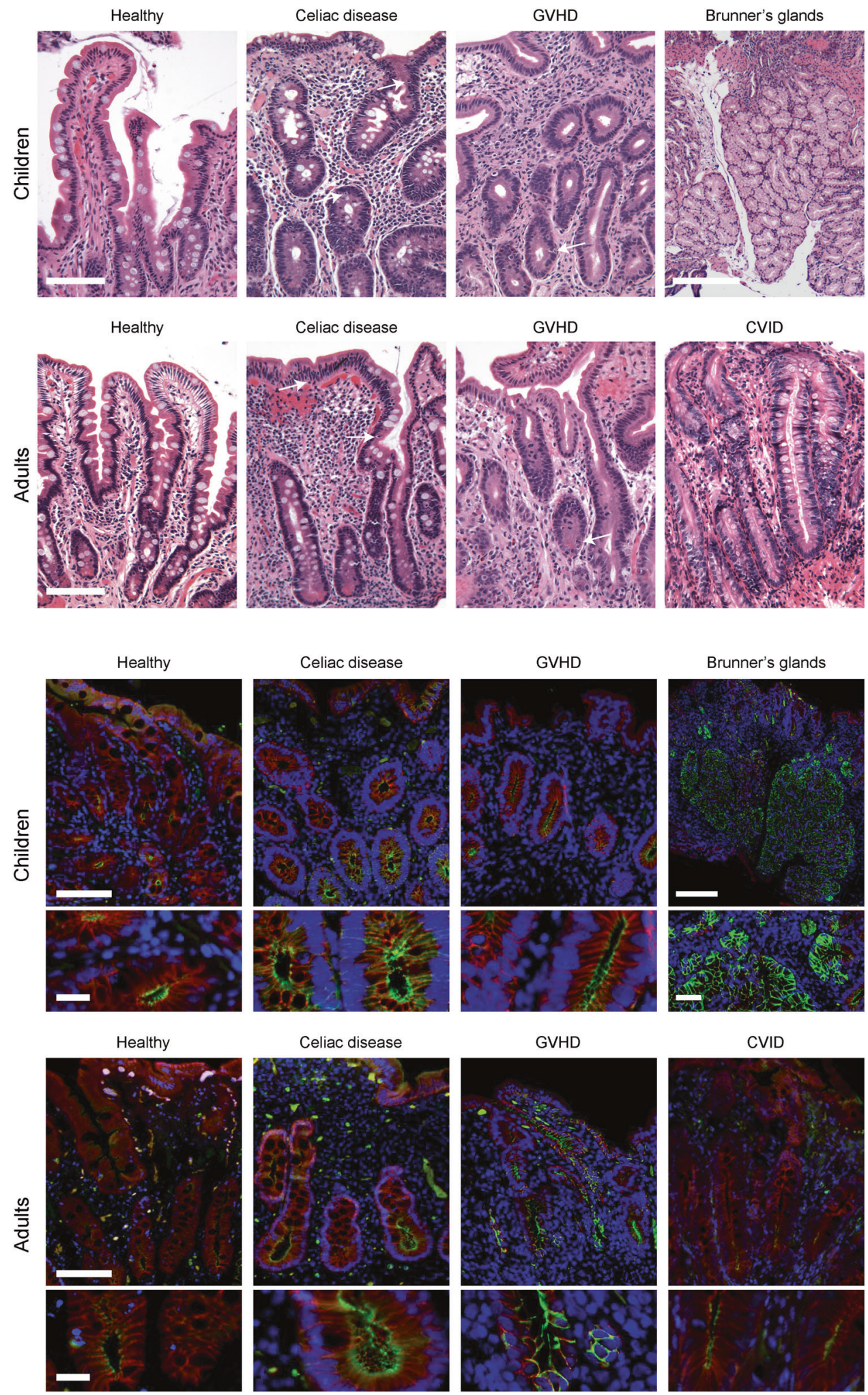

Claudin-2 expression was reduced by $57 \%$ in children with GVHD $(P<0.001$; Fig. 3$)$ but was not significantly changed in adults GVHD patients (Fig. 3).
Only adult cases of CVID were identified, consistent with the natural history of this disease. Despite the dense lymphocytic infiltrates within the lamina propria, claudin-2 


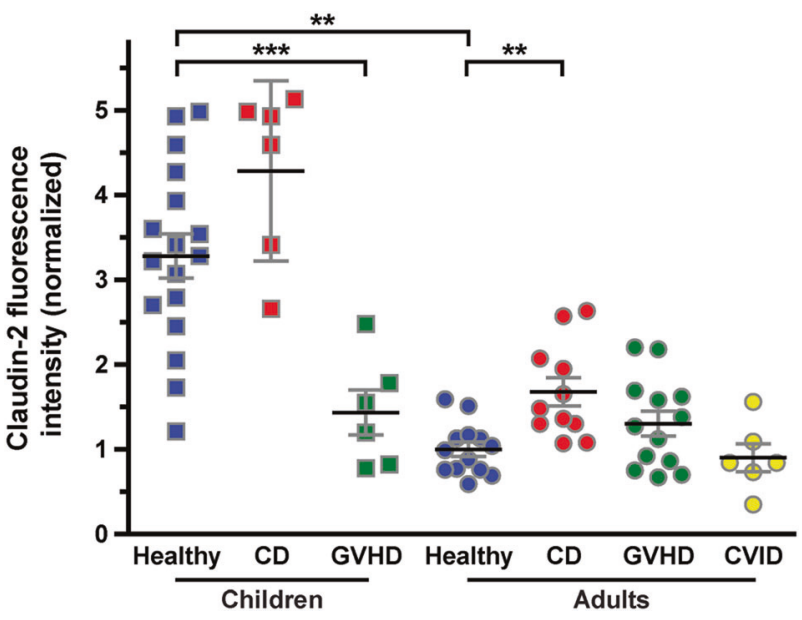

Fig. 3 Quantitative analysis of claudin-2 expression. Claudin-2 fluorescence intensity in children and adults. $n=$ children: 17, 6, 6 (healthy, CD, GVHD); adults: 13, 11, 13, 6 (healthy, CD, GVHD, CVID). **P $<0.005 ; * * * P<0.001$

expression in CVID was similar to that in healthy adults (Fig. 3).

\section{Claudin-15}

The overall distribution of claudin- 15 within villus and crypt epithelial cells of healthy pediatric and adult biopsies was similar to that of claudin-2 (Fig. 4), but claudin-15 expression was greater in adults $(P<0.05$; Fig. 5). Claudin15 expression was not detected in Brunner's glands (Fig. 4).

Claudin-15 expression was not affected by CD in either children or adults (Fig. 5). Claudin-15 expression was, however, significantly reduced in biopsies from adults with GVHD or CVID $(P<0.005$; Fig. 5). As a result, claudin-15 expression in adults with GVHD or CVID was similar to that in healthy, CD, and GVHD children (Fig. 5). Claudin15 expression was unchanged in children with GVHD (Fig. 5).

\section{Discussion}

This study addresses some issues that have been studied previously $[10,11]$, but examines them from significantly different perspectives. First, although claudin-2 and claudin-15 expression within the intestine have been assessed during development, prior analyses were done in mice $[1,7]$. No previous reports have used quantitative morphometry to assess claudin- 2 and claudin- 15 expression in intestinal biopsies from healthy and diseased children and adults.

One previous study assessed claudin-2 and claudin- 15 expression in adults with $\mathrm{CD}$ using western blots of whole biopsies [10]. A separate study assessed the proportion of cells expressing claudin-2 in pediatric $\mathrm{CD}$, but claudin-2 was primarily intracellular in that study [11]. Although total expression of a claudin within the biopsy and the proportion of epithelial cells expressing a claudin can be informative, it is ultimately the number of claudin molecules at the epithelial tight junction that define paracellular permeability. Our analyses of tight junction-associated claudin-2 and claudin-15 therefore provide substantive new insight into the effects of disease on paracellular permeability.

Given the importance of claudin-2 and claudin- 15 to $\mathrm{Na}^{+}$, water, and nutrient transport, further definition of their expression patterns in health and disease is required to understand the pathophysiology of malabsorptive diarrhea. Rather than focusing exclusively on $\mathrm{CD}$, we chose to compare CD with GVHD, another malabsorptive disease resulting from epithelial damage inflicted by cytolytic $\mathrm{T}$ cells $[24,25]$. In adult cases, we also assessed claudin-2 and claudin-15 expression in patients with CVID (which typically presents well after puberty), an uncommon cause of malabsorptive diarrhea.

Our finding that claudin-2 expression is reduced and claudin-15 expression is increased during human development is consistent with immunostain data from mouse jejunum reported by two groups who analyzed expression at either 1 and 28 days or 2 and 8 weeks of life $[1,7]$. The primary difference between our results in normal human children and adults and previous analyses of mice was that we detected expression of both claudins within villous enterocytes at all ages. This may be because the children studied here were at least partially weaned, while the mouse studies focused on times before weaning. Overall, the data demonstrate that claudin-2 downregulation and claudin- 15 upregulation occur during normal human postnatal development and are independent of weaning.

One potential explanation for increased claudin-2 expression in children could be that it provides an advantage over claudin- 15 during rapid growth, where demands on $\mathrm{Na}^{+}$-nutrient cotransport systems are maximized. Recent work showing that claudin-2 and claudin-15 differ biophysically, with the claudin- 2 channel accommodating only dehydrated or partially dehydrated $\mathrm{Na}^{+}$and the larger claudin-15 channel accommodating both water and hydrated $\mathrm{Na}^{+}$[28], may shed further light on this. That work found that water and $\mathrm{Na}^{+}$are competitive inhibitors of one another for flux via the claudin- 15 channel, but that this was not true for claudin-2. We speculate that this difference may allow claudin-2 to be a more efficient conductor of paracellular $\mathrm{Na}^{+}$efflux into the lumen, which could, in turn, enhance $\mathrm{Na}^{+}$, water, and nutrient absorption. This idea is supported by our report that intestinal claudin-2 overexpression increases stool water and $\mathrm{Na}^{+}$in healthy mice 
Fig. 4 Claudin-15 expression. Representative claudin-15 immunofluorescence images for each subject group are shown. Immunofluorescence shows claudin-15 (green), E-cadherin (red), and DNA (blue). Note the lack of claudin-15 expression within Brunner's gland epithelia. Scale bar $=100 \mu \mathrm{m}$; inset bar $=$ $20 \mu \mathrm{m}$. Brunner's gland scale: bar $=250 \mu \mathrm{m}$; inset bar $=50 \mu \mathrm{m}$
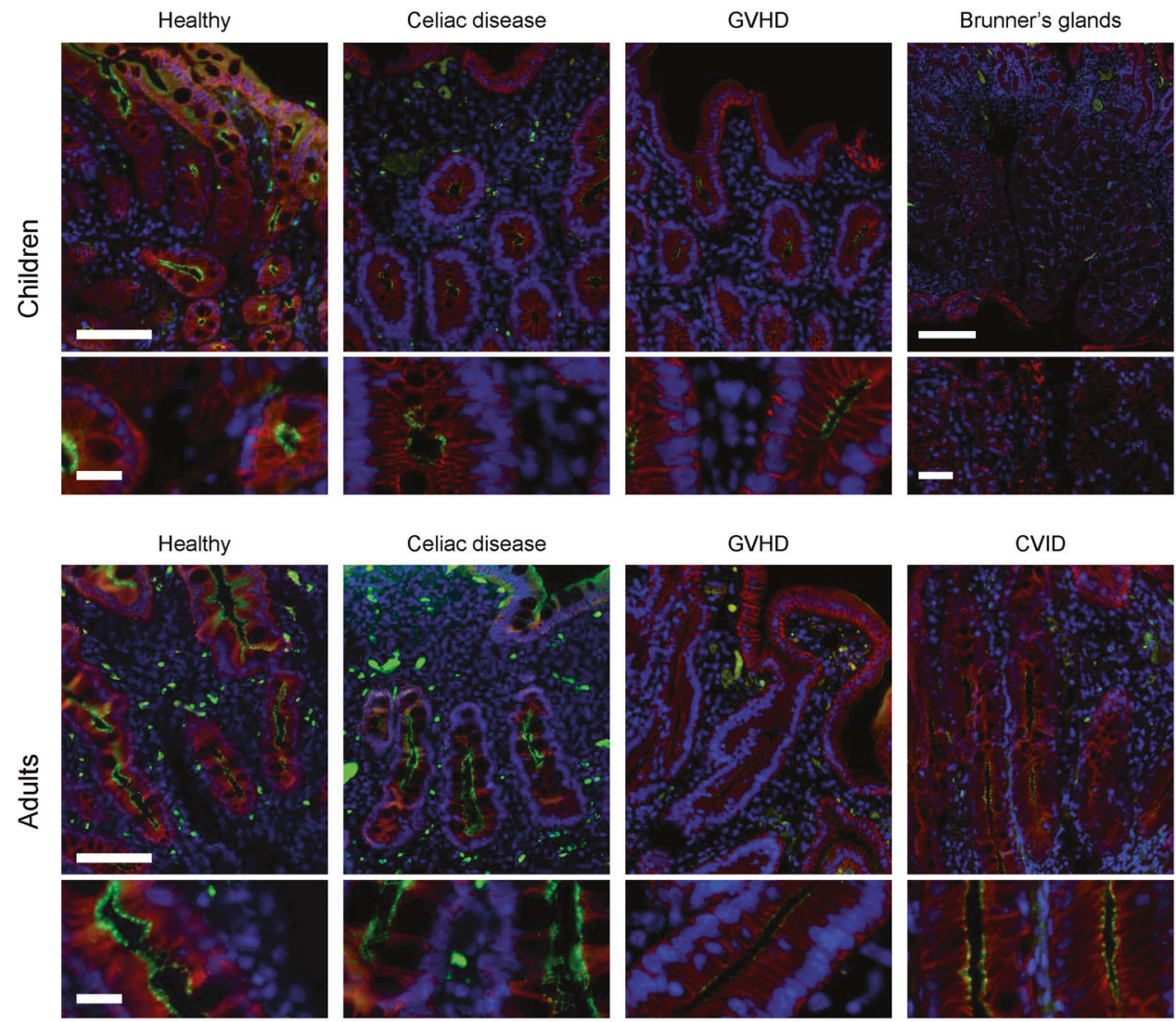

Celiac disease
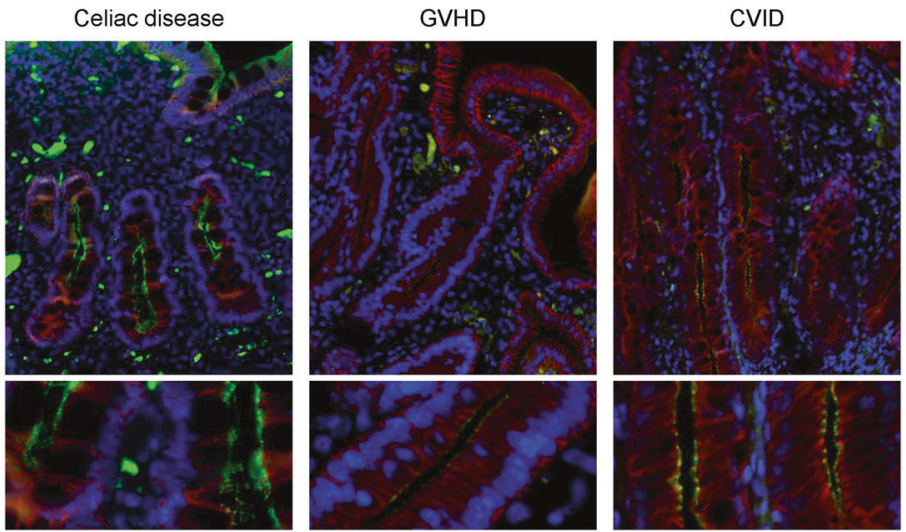

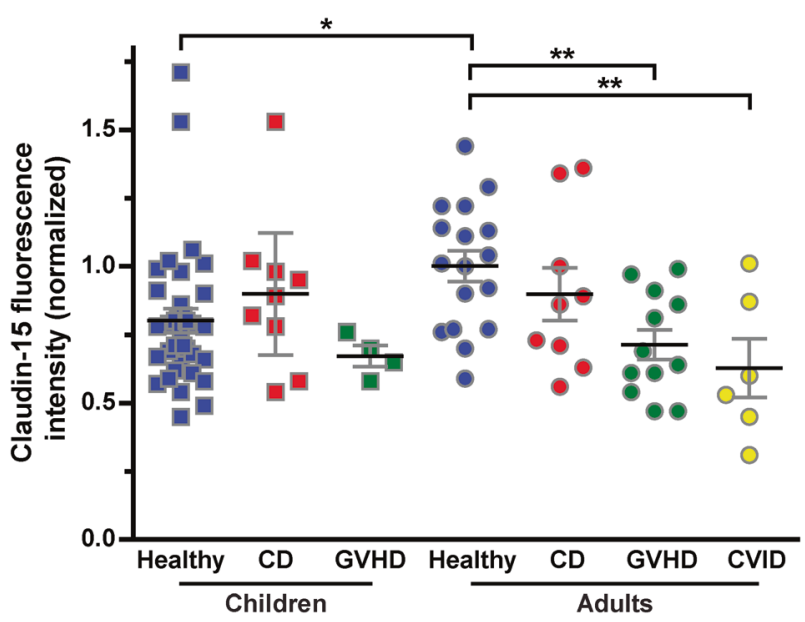

Fig. 5 Quantitative analysis of claudin-15 expression. Claudin-15 fluorescence intensity in children and adults. $n=$ children: $35,9,4$ (healthy, CD, GVHD); adults: 17, 9, 12, 6 (healthy, CD, GVHD, CVID) $* P<0.05 ; * * P<0.005$

[29]. Our observation that claudin-2, but not claudin-15, is highly expressed in Brunner's glands, which secrete bicarbonate-rich fluid to hydrate and neutralize the $\mathrm{pH}$ of gastric contents is also consistent with such a functional difference between these $\mathrm{Na}^{+}$and water pore-forming claudins.
Because our study focused on altered expression patterns in disease, where villi are often absent, the analyses concentrated on claudin expression within the crypt epithelium and specifically analyzed claudin proteins localized at the tight junction. This is an advantage of our study that likely explains the more modest increases in claudin-2 and claudin-15 expression we detected in human CD relative to previous work that depended on western blots that cannot provide spatial resolution at either tissue or subcellular levels [10, 30]. Thus, for claudin-2, which, in adults, is primarily expressed in crypt epithelium, the presence of villous epithelium in western blot specimens may have led to an underestimate of expression. The nearly $200 \%$ increase in claudin- 2 expression in adult $\mathrm{CD}$ reported by that study may therefore be overstated [10]. Our focus on individual intercellular junctions within duodenal crypts eliminated such biases. This could explain why we detected a somewhat smaller $68 \%$ increase in claudin-2 expression in adult $\mathrm{CD}$ than was reported previously. Similar to previous studies [10, 11], we also detected cytoplasmic claudin-2 pools but did not include these in our analyses of tight junction-associated claudin- 2 .

The caveats noted above with regard to comparisons between western blot and quantitative immunofluorescence data also apply to claudin-15. This may explain why we were 
unable to confirm a previous report that claudin-15 expression was upregulated in adult CD [10]. We did, however, find that claudin-15 expression was downregulated in adult, but not pediatric, GVHD. Conversely, claudin-2 was downregulated in pediatric, but not adult, GVHD. This difference highlights the need to consider both the patient population and controls to which patient biopsies are compared when performing analyses such as these and, more importantly, suggests that the pathophysiology of diarrhea in these patient groups may differ. Moreover, the observation that $\mathrm{Na}^{+}$and water poreforming claudins are downregulated in GVHD in both children and adults suggests that mechanisms of altered intestinal paracellular transport are significantly different between CD and GVHD. The ongoing crypt cell damage that characterizes GVHD may be one contributor to these differences.

Given the presumed immune-mediated diarrhea pathophysiology in CVID, we were surprised to find no difference in claudin-2 expression in these biopsies. We had anticipated that the dense lymphocytic infiltrates within the lamina propria in CVID would produce cytokines that increase claudin-2 expression [29, 31, 32]. This was not the case Moreover, claudin-15 expression was reduced. These data suggest that incomplete $\mathrm{Na}^{+}$-nutrient cotransport due to insufficient paracellular $\mathrm{Na}^{+}$recycling may contribute to diarrheal disease in CVID. Although this clearly requires further study, it may be difficult, as CVID remains a relatively uncommon disease despite being frequent among immunodeficiencies.

In summary, these data confirm some, but not all, previous observations in adult CD patients, expand on and refine previous observations in pediatric $\mathrm{CD}$ patients and are the first to compare expression in children and adults. In addition, this is the first report to assess claudin expression in GVHD and CVID. While the sample size was small, the implications for advancing our understanding and ultimately influencing treatment of malabsorptive diarrhea are significant.

Acknowledgements This work was supported by National Institutes of Health (R01DK61631, R01DK68271, R24DK099803), the Harvard Digestive Disease Center (P30DK034854), and the University of Chicago Cancer Center (P30CA014599). AS and JRT were involved in case acquisition and tissue microarray development; MLDM, SPN, SY, and JRT were involved in data acquisition and analysis, figure preparation, and paper creation. All authors approved the final paper.

\section{Compliance with ethical standards}

Conflict of interest The authors declare that they have no conflict of interest.

Publisher's note Springer Nature remains neutral with regard to jurisdictional claims in published maps and institutional affiliations.

\section{References}

1. Tamura A, Hayashi H, Imasato M, et al. Loss of claudin-15, but not claudin-2, causes $\mathrm{Na}+$ deficiency and glucose malabsorption in mouse small intestine. Gastroenterol. 2011;140:913-23.

2. Wada M, Tamura A, Takahashi N, et al. Loss of claudins 2 and 15 from mice causes defects in paracellular $\mathrm{Na}+$ flow and nutrient transport in gut and leads to death from malnutrition. Gastroenterol. 2013;144:369-80.

3. Muto S, Hata M, Taniguchi J, et al. Claudin-2-deficient mice are defective in the leaky and cation-selective paracellular permeability properties of renal proximal tubules. Proc Natl Acad Sci USA. 2010;107:8011-6.

4. Tamura A, Kitano Y, Hata M, et al. Megaintestine in claudin-15deficient mice. Gastroenterol. 2008;134:523-34.

5. Turner JR, Black ED. NHE3-dependent cytoplasmic alkalinization is triggered by $\mathrm{Na}(+)$ - glucose cotransport in intestinal epithelia. Am J Physiol Cell Physiol. 2001;281:C1533-1541.

6. Lin R, Murtazina R, Cha B, et al. D-glucose acts via sodium/ glucose cotransporter 1 to increase NHE3 in mouse jejunal brush border by a $\mathrm{Na}+/ \mathrm{H}+$ exchange regulatory factor 2 - dependent process. Gastroenterol. 2011;140:560-71.

7. Holmes JL, Van Itallie CM, Rasmussen JE, et al. Claudin profiling in the mouse during postnatal intestinal development and along the gastrointestinal tract reveals complex expression patterns. Gene Expr Patterns. 2006;6:581-8.

8. Weber CR, Nalle SC, Tretiakova M, et al. Claudin-1 and claudin2 expression is elevated in inflammatory bowel disease and may contribute to early neoplastic transformation. Lab Investig. 2008;88:1110-20.

9. Luettig J, Rosenthal R, Barmeyer C, et al. Claudin-2 as a mediator of leaky gut barrier during intestinal inflammation. Tissue Barriers. 2015;3:e977176.

10. Schumann M, Gunzel D, Buergel N, et al. Cell polaritydetermining proteins Par-3 and PP-1 are involved in epithelial tight junction defects in coeliac disease. Gut. 2012;61:220-8.

11. Szakal DN, Gyorffy H, Arato A, et al. Mucosal expression of claudins 2, 3 and 4 in proximal and distal part of duodenum in children with coeliac disease. Virchows Arch. 2010;456:245-50.

12. Kohout P. Small bowel permeability in diagnosis of celiac disease and monitoring of compliance of a gluten-free diet (gut permeability in celiac disease). Acta Medica. 2001;44:101-4.

13. Nalle SC, Kwak HA, Edelblum KL, et al. Recipient NK cell inactivation and intestinal barrier loss are required for MHCmatched graft-versus-host disease. Sci Transl Med. 2014;6:243ra287.

14. Nalle SC, Turner JR. Intestinal barrier loss as a critical pathogenic link between inflammatory bowel disease and graft-versus-host disease. Mucosal Immunol. 2015;8:720-30.

15. Koltun WA, Bloomer MM, Colony $\mathrm{P}$, et al. Increased intestinal permeability in rats with graft versus host disease. Gut. 1996;39:291-8.

16. Green PH, Jabri B. Celiac disease. Annu Rev Med. 2006;57:207-21.

17. Guandalini S, Assiri A. Celiac disease: a review. JAMA Pediatr. 2014;168:272-8.

18. Green PH, Cellier C. Celiac disease. N Engl J Med. 2007;357:1731-43.

19. Diosdado B, van Bakel H, Strengman E, et al. Neutrophil recruitment and barrier impairment in celiac disease: a genomic study. Clin Gastroenterol Hepatol. 2007;5:574-81.

20. Calleja S, Vivas S, Santiuste M, et al. Dynamics of nonconventional intraepithelial lymphocytes-NK, NKT, and gammadelta T-in celiac Disease: relationship with age, diet, and histopathology. Dig Dis Sci. 2011;56:2042-9. 
21. McAllister CS, Kagnoff MF. The immunopathogenesis of celiac disease reveals possible therapies beyond the gluten-free diet. Semin Immunopathol. 2012;34:581-600.

22. Cogbill $\mathrm{CH}$, Drobyski WR, Komorowski RA. Gastrointestinal pathology of autologous graft-versus-host disease following hematopoietic stem cell transplantation: a clinicopathological study of 17 cases. Mod Pathol. 2011;24:117-25.

23. Sivula J, Cordova ZM, Tuimala J, et al. Toll-like receptor gene polymorphisms confer susceptibility to graft-versus-host disease in allogenic hematopoietic stem cell transplantation. Scand J Immunol. 2012;76:336-41.

24. Washington K, Jagasia M. Pathology of graft-versus-host disease in the gastrointestinal tract. Hum Pathol. 2009;40:909-17.

25. Sung D, Iuga AC, Kato T, et al. Crypt apoptotic body counts in normal ileal biopsies overlap with graft-versus-host disease and acute cellular rejection of small bowel allografts. Hum Pathol. 2016;56:89-92.

26. Daniels JA, Lederman HM, Maitra A, et al. Gastrointestinal tract pathology in patients with common variable immunodeficiency (CVID): a clinicopathologic study and review. Am J Surg Pathol. 2007;31:1800-12.
27. Washington K, Stenzel TT, Buckley RH, et al. Gastrointestinal pathology in patients with common variable immunodeficiency and X-linked agammaglobulinemia. Am J Surg Pathol. 1996;20:1240-52.

28. Rosenthal R, Gunzel D, Piontek J, et al. Claudin-15 forms a water channel through the tight junction with distinct function compared to claudin-2. Acta Physiol. 2019;12:e13334.

29. Tsai PY, Zhang B, He WQ, et al. IL-22 upregulates epithelial claudin-2 to drive diarrhea and enteric pathogen clearance. Cell Host Microbe. 2017;21:671-81 e674.

30. Schumann M, Siegmund B, Schulzke JD, et al. Celiac Disease: role of the epithelial barrier. Cell Mol Gastroenterol Hepatol. 2017;3:150-62.

31. Heller F, Florian P, Bojarski C. et al. Interleukin-13 is the key effector Th2 cytokine in ulcerative colitis that affects epithelial tight junctions, apoptosis, and cell restitution. Gastroenterology. 2005;129:550-64.

32. Weber CR, Raleigh DR, Su L, et al. Epithelial myosin light chain kinase activation induces mucosal interleukin-13 expression to alter tight junction ion selectivity. J Biol Chem. 2010;285: 12037-46. 